\title{
Power System Simulation and Preliminary Modeling Based on the Smart Grid Core Standards
}

\author{
Yue $\mathrm{ZHAO}^{1,}$, , Jingjin $\mathrm{CAO}^{2, \mathrm{~b}}$ \\ ${ }^{1}$ State Grid Taigu Power Company, State Grid Jinzhong Power Company, Jinzhong, 030800, China \\ 2Equipment Status Evaluation Center, State Grid Shanxi Electrical Power Research Institute, \\ Taiyuan, 030001, China \\ aemail: 547491767@qq.com, bemail:caojingjin0123@163.com
}

Keywords: IEC61970; CIM; UML; XML; Analysis of XML; Power system modeling.

\begin{abstract}
With the development of computer technology and the rapid prevalence of the Internet Power started to use computer to realize the unification and standardization. So IEC puts forward IEC 61970 specification series, which makes EMS normalize and interconnects different EMS.CIM has been defined by IEC61970 which commented by UML. Meanwhile, in order to further the support of information exchange on electricity, power industry has developed CIM / XML, which is method used XML to represent the CIM. This article briefly describes the CIM, XML and UML, the exploration of XML document in detail for expression framework is showed in the CIM class diagrams, and complete the parse of XML documents.
\end{abstract}

\section{Introduction}

Smart grid core standards include IEC850, IEC61970 and IEC61968 and so on. IEC850 is an international standard for intelligent substation system. IEC61970 is an international standard for energy management system of power system application program interface[1,2]. IEC61968 is an international standard for energy management system of power distribution system application program interface. Substation configuration file, transmission and distribution system of resource description file are generated based on these standards, contains all the information of power system's composition, structure, protection and control device. This article explores from the file parsing the required data and information related to power system simulation, and sets up power system branch - node raw data model. In the process, we establish the UML framework by using $\mathrm{C}++$ as a bridge, parse the XML file under IEC61970 core standard-CIM code, and establish the node admittance matrix, in order to facilitate people to check and use the data.

\section{CIM model}

In the CIM model, the object-oriented thought provides a new thinking and new method to the power system modeling[3]. Compared to a process oriented way of modeling, CIM description method has advantages in the establishment of the system[4]: the structure of class is clear; the accretion and modification is very convenient. In CIM, the properties and methods of power system resources have made detailed description, according to these methods, it is easy to establish power system model in $\mathrm{C}++$. After a model is established, we parse an XML document. In the article, persistence of the power system model is a emphasis and difficulty, and needs to consider many aspects. Firstly, we establish CIM classes, and then build whole method to find any power system resources from the root node of the CIM sense geographical area. According to discrete power-saving switch model, we establish a zero impedance power saving and bus bar model, finally set up system admittance matrix.

\section{XML parsing process}

The article takes opencim3bus. XML as an example to state the parsing process.

Root node of the XML is RDF, there are two name spaces: RDF space and CIM space. 
RDF has the child nodes including power system resources nodes and comment nodes, the former belongs to the common information model CIM space, its id belongs to the resource description framework space to locate the resource location.

Power system resource node has child nodes to save the attributes of the resources, the content of the child nodes saves the resource of attribute values. Child nodes also belong to the CIM space.

Using MSXML to parse XML document on this basis[5]. Resolution of some key process is as follows:

In $\mathrm{C}++$ program, the root node $\mathrm{p}$-Root element is the key to manipulate XML as the entry of the document. Through the child nodes members of root node the root node list p-Node List can be get, on this basis, the information of each node can be get.

1)The extraction of nodes

List of nodes p-Node list provide function of child nodes according to the index search. The length of p-Node list is obtained at first, various nodes can be get on the basis of cycle length.

2)The node processing

Node contains all the information from the node names which are stored in the character variables node name, and can be created in different kinds by judging the node name.

3)The contact Establishing

In each class, the connection between the classes should be established, these connections can be linked through a unique ID to identify each device, set up some function which accept ID as input parameters, through search, a pointer of the ids instance class is returned.

\section{The establishment of the node admittance matrix:}

The actual power system is a complex large system, it includes hundreds or even thousands of bus bar, and the network structure is complex, its power flow calculation must use the computer. And the calculation of the tidal current is generally includes the following steps: establishing the mathematical model of power flow calculation; determining the calculation method of solving mathematical model; setting calculation flow chart of calculation program; debugging and running the application. Then we should analysis and study the calculation results and examine the calibration procedure, it includes the preparation and entry of original data, power flow calculation, the calculation results analysis, etc.

Electric power network's mathematical model describes running state of electric power network, the interrelation between the variables and the network parameters, and reflects the state of the network, the performance of the mathematical equations, namely the electricity network equations. And power network equation has the node voltage equation and loop current equation and cut set voltage equation, etc. If writing these equations, the most important thing is to know that the power system network node admittance matrix. The node admittance matrix of the diagonal elements in each row has only 3 5 a nonzero elements on average, the other are zero elements, so the admittance matrix of the sparse degree is high, but in programming, using the sparse can greatly save storage unit and improve the calculation speed.

\section{Test results}

We analysis XML document and establish the admittance matrix according to required information. Firstly, the base class and large number of contact are established; secondly, the XML file is parsed, and the primary information is stored; thirdly, XML information is reprocessed, the node admittance matrix is established and finally output. The final result is shown in Fig.1(exe format): 


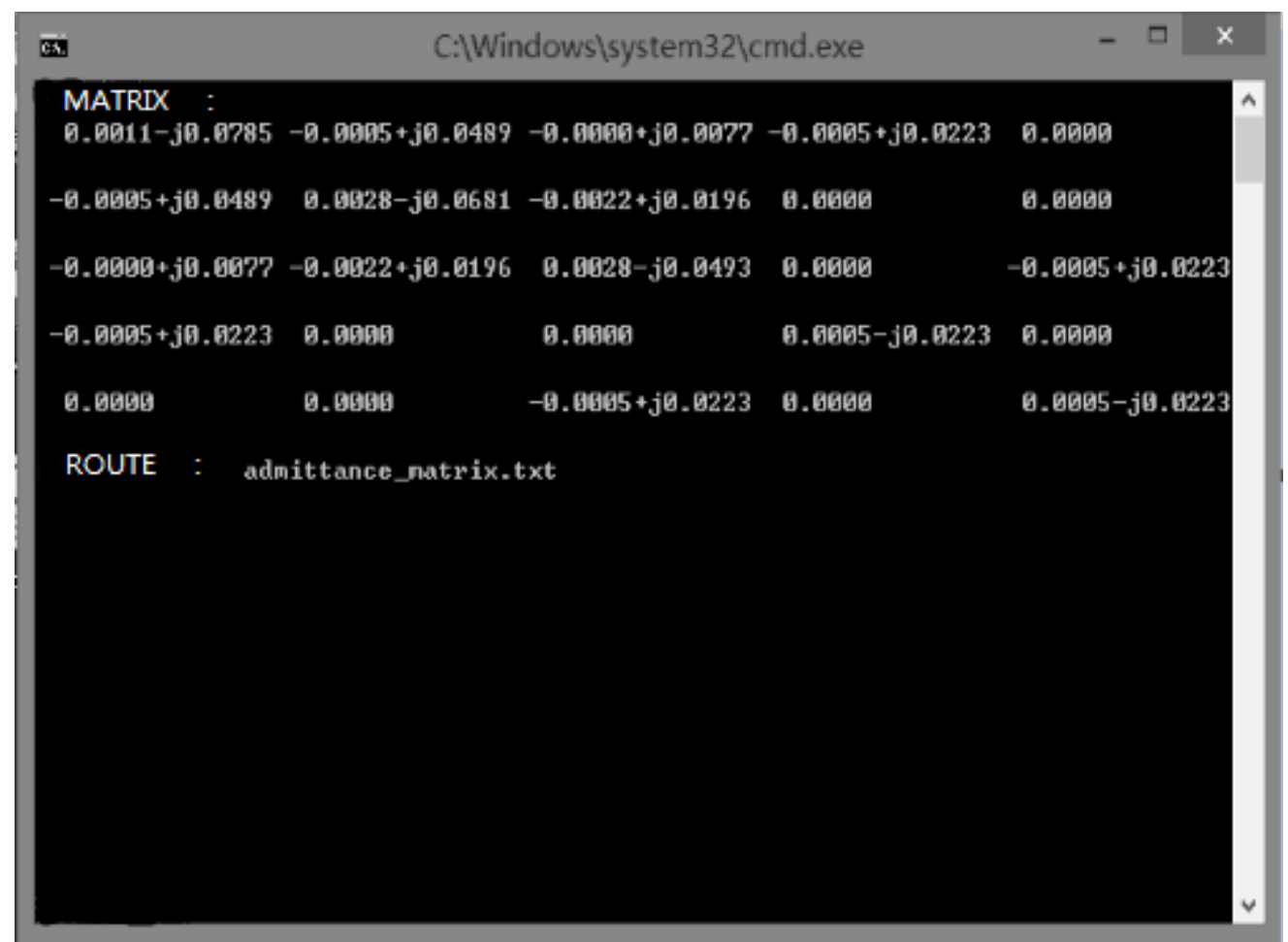

Fig.1. The final result of the node admittance matrix

The parse XML file system diagram is shown in Fig.2.

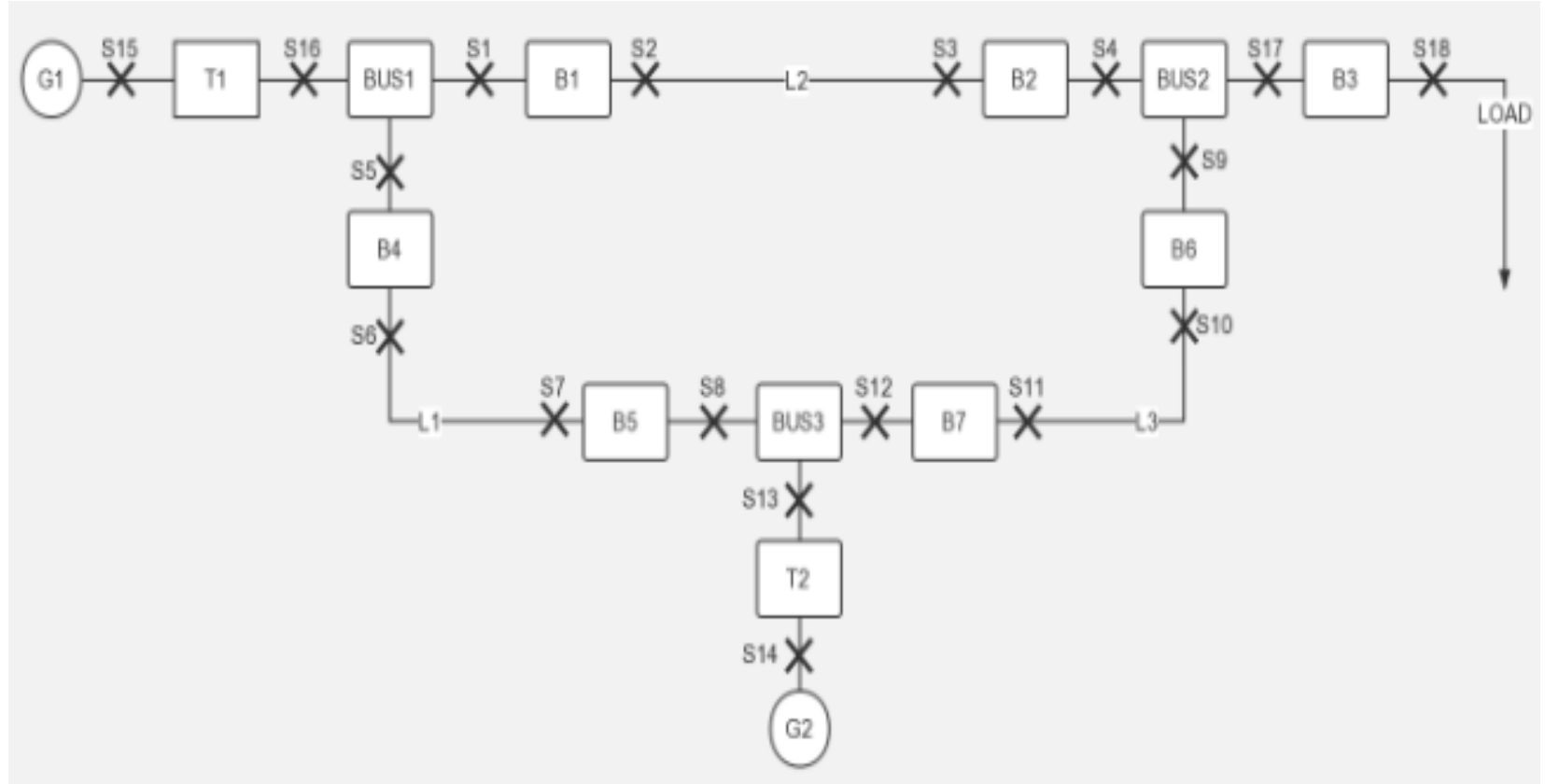

Fig.2. The parse XML file system diagram

Bus bar graph is shown in Fig.3. 


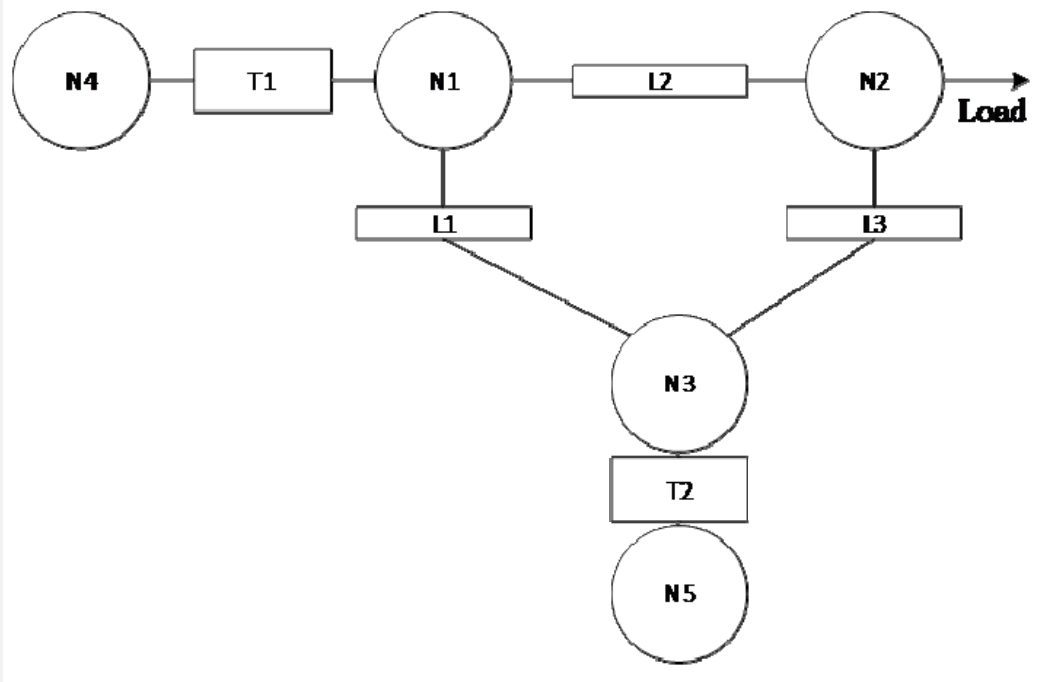

Fig.3. Bus bar graph

As is shown in Fig. 3 , the system is actually a 5 node power system network, there are 3 wires substation linked by wires, two of the power supplies are connected to the two substations by transformers, the left is connected to zero impedance load.

The mutual admittance matrix of system can be obtained: The first line in the first column of mutual admittance matrix element represents the admittance to the ground in node N1, the first line of the second column element represents the mutual admittance between node $\mathrm{N} 1$ and $\mathrm{N} 2$, it is the same with the other nodes. So the admittance matrix is obtained according to the admittance and mutual admittance.

\section{Conclusion}

This article briefly describes the CIM, XML and UML, the exploration of XML document in detail for expression framework is showed in the CIM class diagrams, and complete the parse of XML documents. After further perfect, the result have a chance to become the power industry dedicated XML parser conformed to the rules of CIM.

\section{References}

[1] Zhang Shenming. Liu Guoding. IEC 61970 Standard Series [J]. Electric Power Automation, $2002(14)$.

[2] Zhang Shenming. Bu Fanqiang. Yao Jianguo,et al. Follow the IEC 61970 standard real-time database management system [J]. Electric Power Automation, 2002 (1).

[3] Wang Mingkun. Power system research of CIM model description and data exchange realization [D].Sichuan University:2004,5.

[4] Wang Zhanli. Using MSXML to parse XML files[J]. Scientific and Technological Information, 2009(15).

[5] Wang Lei. Mi Zengqiang. Ren Hui, et al. Research of the power system model based on CIM XML [J]. China Electric Power Education, 2006. 